\title{
NATURAL RADIOACTIVITY OF WESTERN ANATOLIAN PLUTONS, TURKEY
}

\author{
Papadopoulos A. ${ }^{1}$, Altunkaynak S. ${ }^{2}$, Koroneos A. ${ }^{1}$, Unal A. ${ }^{2}$ and Kamaci O. ${ }^{2}$ \\ ${ }^{1}$ Department of Mineralogy, Petrology and Economic Geology, School of Geology, Aristotle \\ University of Thessaloniki, 54124 Thessaloniki, Greece, koroneos@geo.auth.gr, \\ argpapad@geo.auth.gr \\ ${ }^{2}$ Department of Geological Engineering, Faculty of Mines, Istanbul Technical University, 34469 \\ Maslak, Istanbul,Turkey,safak@itu.edu.tr,alp.unal@itu.edu.tr,kamaciom@itu.edu.tr
}

\begin{abstract}
The natural radioactivity of the Western Anatolian plutonic bodies (Turkey), as well as the assessment of any potential health hazard due to their usage as decorative building materials is studied. Seventy samples from Western Anatolian plutonic bodies, including various rock-types from quartz-monzodiortie to syenogranite, have been measured for their natural radioactivity using $\gamma$-spectrometry. According to the experimental results the natural radioactivity levels were ranged up to $229.62 \mathrm{~Bq} . \mathrm{kg}$ ${ }^{1}$ for ${ }^{226} \mathrm{Ra}$, up to $207.32 \mathrm{~Bq} \cdot \mathrm{kg}^{-1}$ for ${ }^{232} \mathrm{Th}$ and up to $2541.95 \mathrm{~Bq} \cdot \mathrm{kg}^{-1}$ for ${ }^{40} \mathrm{~K}$, with a mean value of 57.67 ( \pm 38.13$), 80.30$ ( \pm 42.00$)$ and 1071.92 ( \pm 405.24$)$ Bq. $\mathrm{kg}^{-1}$ respectively, which are below the international representative mean values for granite stones. The increment on the external $\gamma$-radiation effective dose rate appears a mean value of $0.27( \pm 0.19) \mathrm{mSv} \cdot \mathrm{y}^{-1}$, scattering below $1 \mathrm{mSv} \cdot \mathrm{y}^{-1}$. In case of the internal $\alpha$-radiation a mean value of $0.14( \pm 0.10) \mathrm{mSv} \cdot \mathrm{y}^{-1}$, scattering below $0.5 \mathrm{mSv} . \mathrm{y}^{-}$ ${ }^{1}$ was estimated. The majority of the samples increase the external and the internal dose less than $30 \%$ of the maximum permitted limit of the effective dose rate. Therefore, at least from radiological point of view, the plutonic rocks of Western Anatolia could be safely used as decorative building materials.

Keywords: Building materials, External-Internal exposure, Radiation Index.
\end{abstract}

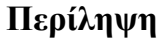

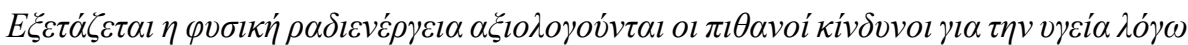

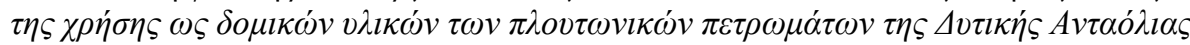

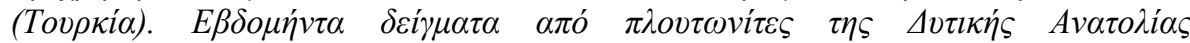

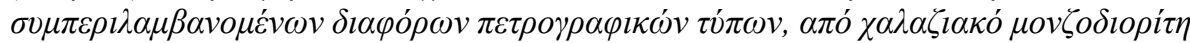

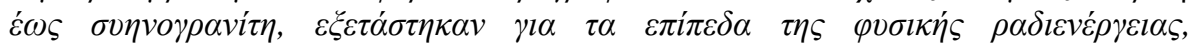

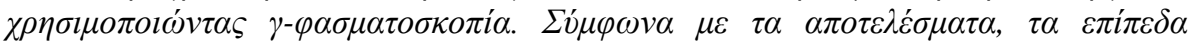

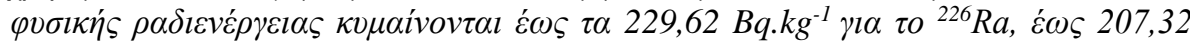

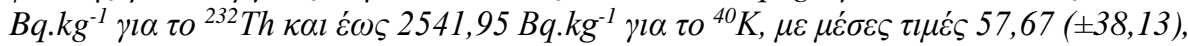

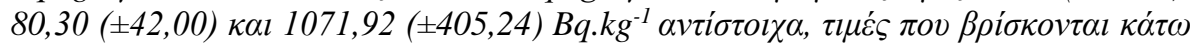

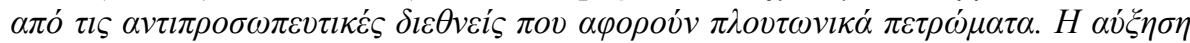

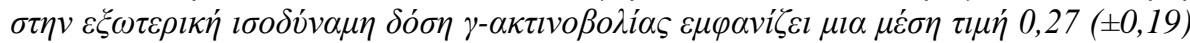

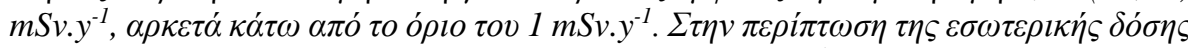

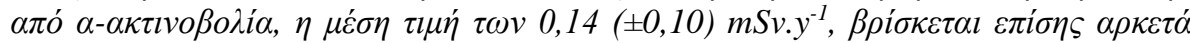

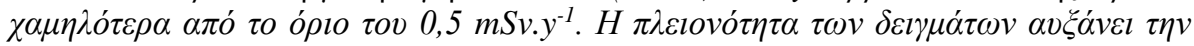




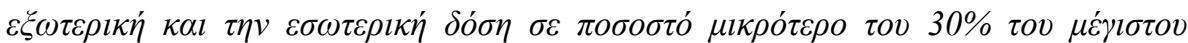

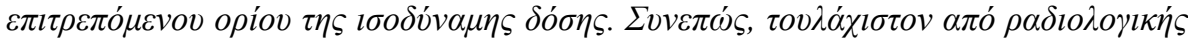

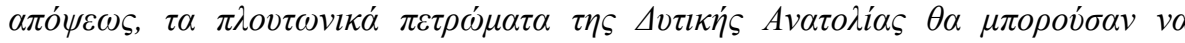

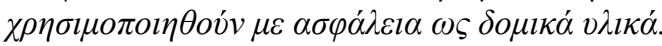

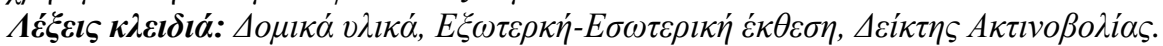

\section{Introduction}

Plutonic rocks (gabbro to granite) are widely used as decorative building materials due to their durability and appearance. These rocks, due to their mineralogical composition, are likely to contain high concentrations of natural radionuclides. The purpose of setting controls on the radioactivity of building materials is to limit the radiation exposure due to materials with enhanced or elevated levels of natural radionuclides.

Several works present in the literature, are referred to radiation risks of granite used as decorative building material (Papadopoulos et al., 2013 and references therein), while several authors have studied the natural radioactivity of plutonic bodies of Turkey e.g. Örgün et al. (2007). In the present work, the natural radioactivity of the major Western Anatolian plutonic bodies in Turkey as well as the assessment of any potential health hazard in case they were used as decorative building materials are studied.

\section{Materials and Methods}

\subsection{Geological Setting}

The Cenozoic geology of western Anatolia (Turkey) is characterized by intensive magmatic activity producing volcanic and plutonic rocks that can be used as decorative building materials (Fig. 1). Nature, origin and tectonic setting of these magmatic rocks have been studied in detail previously by various researchers (i.e. Yılmaz, 1989; Güleç, 1991; Altunkaynak and Yılmaz, 1998; Aldanmaz et al., 2000; Okay and Satır, 2000, 2006; Köprübaş1 and Aldanmaz, 2004; Altunkaynak and Dilek, 2006, Altunkaynak, 2007; Dilek and Altunkaynak, 2007; Altunkaynak and Genç, 2008; Boztuğ et al., 2009; Ersoy et al., 2009; Hasözbek et al., 2010; Altunkaynak et al., 2012a,b; Erkül and Erkül, 2012; Erkül, 2010).

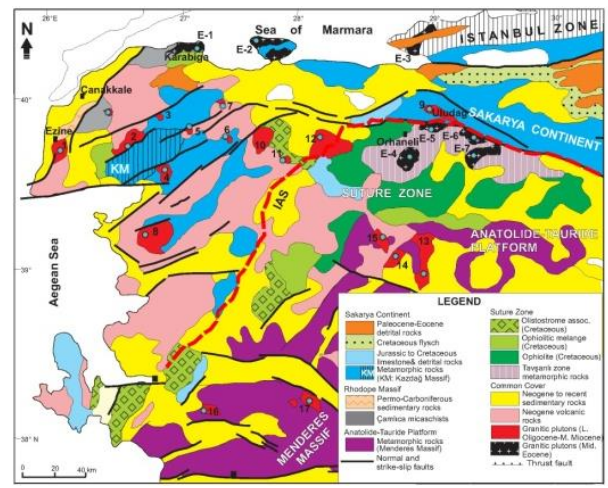

Figure 1 - Simplified geological map of western Anatolia showing the distribution of Granitoids (Modified from Yılmaz et al., 2000; Okay and Satır, 2006 and Altunkaynak et al., 2012a). IAS; Izmir-Ankara-Erzincan suture zone. E1 to 7: Eocene granitoids (E1: Karabiga, E2: Kapıdağ, E3: Fıstıklı, E4: Orhaneli, E5:Yopuk, E6:Göynükbelen, E7: Gürgenyayla), 1 to 15: Oligo-Miocene granitoids (1- Kestanbol 2-Evciler 3-Hıdırlar-Katrandag 4-Eybek 5Yenice 6-Danisment, 7-Sarıoluk, 8-Kozak 9-Uludag 10, Ilica-Samli 11-Davutlar, 12-Çataldag 13-Egrigoz 14-Koyunaoba 15-Çamlik 16-Turgutlu 17-Salihli granitoids). 
Western Anatolia and adjacent regions (Greece and Bulgaria) are situated along the eastern continuation of the Alpine collisional belt and are affected dominantly by convergent tectonics preceding current extensional tectonics. The final demise of the northern branch of Neo-Tethyan Ocean at a subduction zone dipping northwards beneath the Sakarya continent resulted in a continent-continent collision between the Sakarya and Anatolide-Tauride continental fragments during the Late Cretaceous-pre-Eocene. (Şengör and Yılmaz, 1981; Yılmaz, 1989; Güleç, 1991; Harris et al., 1994). The Izmir-Ankara Suture Zone (IASZ) represents the collision zone between the Anatolide-Tauride platform (ATP) in the south and the Sakarya continent (SC) in the north.

Following the closure of the Neo-Tethyan Ocean, two major magmatic episodes producing granitic plutons occurred within the Cenozoic evolution of western Anatolia. The first episode of postcollisional magmatism developed during the early-late Eocene, and produced mainly medium to highK calc-alkaline, I-type granitoid plutons and associated extrusive rocks (Harris et al., 1994; Koprubasi and Aldanmaz, 2004; Altunkaynak, 2007; Altunkaynak et al., 2012a). The Eocene granitic plutons occur within and north of the IASZ. Among these, Orhaneli, Topuk, Gürgenyayla plutons exposed along the IASZ and are intruded into the Cretaceous blueschist rocks and overlying ophiolitic units. They range in composition from quartz diorite, granodiorite to syenite. Kapıdağ, Fistıklı (Armutlu), Karabiga plutons, on the other hand, crop out along the southern margin of the Marmara Sea. These plutons are intruded into the basement rocks of Sakarya continent north of the IASZ and are composed of monzogranite, granodiorite and granite. The Eocene granitic and volcanic rocks are rare and restricted to NW Anatolia. The following magmatic phase occurred during the late Oligocene and middle Miocene and is known to have produced the widespread granitic plutons (i.e., Kozak, Evciler, Cataldag, Kestanbol, Ilica, Eybek, Egrigoz, Çamlık, Uludağ) and volcanic rocks in western Anatolia (Yilmaz, 1989; Altunkaynak et al., 2012b; Yilmaz, et al., 2001; Ozgenc and Ilbeyli, 2008; Akay 2009). They are represented mostly by medium to high-K calc-alkaline to shoshonitic I-type granitic plutons emplaced into the continental blocks on both side of the IASZ. The Kozak, Evciler, Ilica, Eybek, Çataldağ, granites are the representatives of the granites that were emplaced into the metamorphic basement rocks of the Sakarya continent. The Çamlık and Eğrigöz plutons, on the other hand, were emplaced into the Anatolide-Tauride Platform (i.e. the metamorphic rocks of the Menderes Massif). Late Oligocene- Middle Miocene granitoid plutons and associated volcanic rocks are widespread in the entire west Anatolia (Yılmaz, 1989; Altunkaynak and Dilek, 2006; Altunkaynak et al., 2012b; Erkül and Erkül, 2012; Erkül, 2010). Most of the late Oligocene-middle Miocene granites are represented by caldera type, shallow level intrusions showing close relationships with their co-genetic volcanic rocks in time and space (Yılmaz, 1989; Altunkaynak and Yilmaz, 1998, 1999; Genç, 1998; Yilmaz et al., 2001).

\subsection{Gamma-ray spectroscopy}

The measurements of activity concentrations were undertaken in Low Level Radioactivity Measurement Laboratory in the Istanbul Technical University Energy Institute by using copper lined lead shielding $(10 \mathrm{~cm})$ detector (GAMMA-X HPGe coaxial n-type germanium detector, $45.7 \%$ efficiency and $1.84 \mathrm{keV}$ full width at half maximum for $1.3 \mathrm{MeV}$ of 60Co) with the integrated digital gamma spectrometer (DSPEC jr. 2.0). Statistical confidence level and range were adjusted to $2 \sigma$ and $8 \mathrm{~K}$, respectively. In order to make the energy and efficiency calibration of the gamma spectroscopy system that are necessary for activity determination, the certificated multiple gamma ray emitting large volume source standard was used; including $\mathrm{Am}^{241}, \mathrm{Cs}^{137}, \mathrm{Co}^{60}, \mathrm{~Pb}^{210}, \mathrm{Cd}^{109}, \mathrm{Co}^{57}, \mathrm{Ce}^{139}, \mathrm{Hg}^{203}$, $\mathrm{Sn}^{113}, \mathrm{Sr}^{85}, \mathrm{Y}^{88}$ radioisotopes in the sand matrix in Marinelli geometry as $500 \mathrm{~mL}$ volume, with a density of $1.7 \mathrm{~g} \mathrm{~cm}^{-3}$ and an activity of $1 \mu \mathrm{Ci}$. Samples and standard in Marinelli beakers were counted at the top of the detector. Counting times were adjusted to 15 to $24 \mathrm{~h}$. Peak areas were determined by using GAMMA VISION-32 software program. After measurements, standards and samples were corrected for decay time and mass. 


\subsection{Major elements}

The whole-rock powders were split from 1 to $5 \mathrm{~kg}$ of crushed rocks. Chemical compositions of the samples were determined by using Spectro Ciros Vision ICP-ES for major oxides is given in Table 1.

Table 1 - Major element content (\% w.t.) of the samples (*Altunkaynak et al., 2012a, b).

\begin{tabular}{|c|c|c|c|c|c|c|c|c|c|c|c|c|}
\hline & $\mathrm{SiO}_{2}$ & $\mathrm{TiO}_{2}$ & $\mathrm{Al}_{2} \mathrm{O}_{3}$ & $\mathrm{Fe}_{2} \mathrm{O}_{3}$ & $\mathrm{MnO}$ & $\mathrm{MgO}$ & $\mathrm{CaO}$ & $\mathrm{Na}_{2} \mathrm{O}$ & $\mathrm{K}_{2} \mathrm{O}$ & $\mathrm{P}_{2} \mathrm{O}_{5}$ & LOI & Sum \\
\hline AS209 & 65.17 & 0.47 & 15.41 & 4.45 & 0.08 & 1.96 & 4.08 & 3.23 & 3.68 & 0.19 & 0.90 & 99.62 \\
\hline AS211 & .09 & 0.40 & 15.42 & 3.82 & 0.10 & 1.54 & 3.90 & 3.45 & \begin{tabular}{|l|}
3.11 \\
\end{tabular} & 0.15 & 0.70 & 99. \\
\hline AS234 & 31 & 0.47 & 15.20 & 4.56 & 0.09 & 2.09 & 4.56 & 3.33 & 3.13 & 0.21 & 0.70 & 99. \\
\hline AS236 & 1.26 & 0.49 & 15.94 & 4.42 & 0.08 & 2.08 & 4.43 & 3.37 & 3.67 & 0.19 & \begin{tabular}{|l|}
0.70 \\
\end{tabular} & 99 . \\
\hline AS238 & 3.00 & 0.50 & 16.60 & 4.88 & 0.10 & 1.95 & 4.82 & 3.59 & \begin{tabular}{|l|}
2.94 \\
\end{tabular} & 0.22 & 1.10 & 99. \\
\hline AS239 & 2.42 & 0.57 & 15.84 & 5.42 & 0.11 & 2.68 & 5.01 & 3.42 & \begin{tabular}{|l|}
2.91 \\
\end{tabular} & 0.18 & 1.10 & 99.6 \\
\hline AS240 & 62.71 & 0.52 & 16.16 & 4.78 & 0.10 & 2.25 & 4.51 & 3.38 & \begin{tabular}{|l|}
3.18 \\
\end{tabular} & 0.16 & 1.90 & 99.6 \\
\hline AS241 & 2.40 & 0.53 & 16.64 & 5.07 & 0.10 & 2.30 & 5.39 & 3.44 & \begin{tabular}{|l|}
2.75 \\
\end{tabular} & 0.20 & 0.80 & 99.6 \\
\hline AS245 & 2.75 & 0.56 & 16.21 & 5.33 & 0.10 & 2.64 & 5.13 & 3.37 & \begin{tabular}{|l|}
2.89 \\
\end{tabular} & 0.16 & \begin{tabular}{|l|} 
\\
\end{tabular} & 99.1 \\
\hline 5248 & .51 & 0.50 & 16.08 & 4.77 & 0.09 & 2.21 & 4.65 & 3.46 & \begin{tabular}{|l|}
3.07 \\
\end{tabular} & 0.17 & 1.20 & 99. \\
\hline AT1 & 3.90 & 0.27 & 15.06 & 3.10 & 0.07 & 0.82 & 2.58 & 3.10 & \begin{tabular}{|l|}
4.22 \\
\end{tabular} & 0.11 & 2.05 & 100. \\
\hline AT2 & 4.51 & 0.03 & 13.68 & 0.63 & 0.16 & 0.05 & 1.08 & 4.43 & 3.56 & $<0.01$ & \begin{tabular}{|l|}
0.70 \\
\end{tabular} & 98.8 \\
\hline AT3 & 8.02 & 0.38 & 14.75 & 3.17 & 0.07 & 0.99 & 2.51 & 3.46 & \begin{tabular}{|l|}
4.04 \\
\end{tabular} & 0.14 & \begin{tabular}{|l|}
1.01 \\
\end{tabular} & 98.5 \\
\hline ÇAT4 & 7.68 & 0.35 & 15.49 & 3.25 & 0.09 & 0.77 & 3.36 & 4.08 & \begin{tabular}{|l|}
2.94 \\
\end{tabular} & 0.17 & 0.70 & 98.8 \\
\hline AT5 & 3.57 & 0.04 & 14.29 & 0.66 & 0.03 & 0.22 & 1.11 & 3.45 & \begin{tabular}{|l|}
4.06 \\
\end{tabular} & 0.08 & 1.89 & 99.4 \\
\hline AT6 & .25 & $\overline{0.04}$ & 13.58 & 0.45 & 0.02 & 0.11 & \begin{tabular}{|l|}
0.82 \\
\end{tabular} & 3.76 & \begin{tabular}{|l|}
3.92 \\
\end{tabular} & 0.06 & 0.86 & 100.8 \\
\hline 8388 & .34 & 0.22 & 15.10 & 1.71 & 0.04 & 0.43 & 2.09 & 3.90 & \begin{tabular}{|l|}
3.17 \\
\end{tabular} & 0.07 & 1.01 & 101.0 \\
\hline OS409 & 2.64 & 0.09 & 14.88 & 0.80 & 0.01 & 0.19 & \begin{tabular}{|l|}
1.12 \\
\end{tabular} & 3.63 & \begin{tabular}{|l|}
5.37 \\
\end{tabular} & 0.09 & 1.10 & 99. \\
\hline ULU3 & .39 & 0.26 & 15.39 & 1.72 & 0.04 & 0.73 & 2.16 & 4.26 & \begin{tabular}{|l|}
2.73 \\
\end{tabular} & 0.12 & 0.90 & 99. \\
\hline ULU5 & 1.08 & 0.27 & 15.65 & 1.56 & 0.02 & 0.52 & 1.75 & 3.97 & \begin{tabular}{|l|}
3.63 \\
\end{tabular} & 0.11 & \begin{tabular}{|l|}
1.10 \\
\end{tabular} & 99.6 \\
\hline LU6 & 1.67 & 0.26 & 15.14 & 1.59 & 0.03 & 0.63 & 2.08 & 4.21 & \begin{tabular}{|l|}
3.20 \\
\end{tabular} & 0.11 & 0.80 & 99.7 \\
\hline LU8 & 1.91 & 0.23 & 15.30 & 1.37 & 0.03 & 0.48 & 1.82 & 4.08 & 3.41 & 0.10 & 1.00 & 99.7 \\
\hline $\mathrm{U} 11$ & .42 & 0.25 & 15.13 & 1.52 & 0.03 & 0.63 & 2.01 & 4.11 & \begin{tabular}{|l|}
3.28 \\
\end{tabular} & 0.11 & 1.30 & 99.7 \\
\hline LU12 & 2.03 & 0.24 & 15.25 & 1.44 & 0.03 & 0.50 & 1.36 & 3.96 & \begin{tabular}{|l|}
3.91 \\
\end{tabular} & $\begin{array}{ll}0.13 \\
\end{array}$ & 0.90 & 99. \\
\hline YB10 & 58.26 & 0.68 & 17.53 & 6.90 & 0.14 & 3.05 & 6.96 & 3.91 & \begin{tabular}{|l|}
1.61 \\
\end{tabular} & 0.17 & 0.50 & 99. \\
\hline EYB14 & 60.41 & 0.69 & 16.22 & 6.50 & 0.13 & 3.00 & 5.39 & 3.75 & \begin{tabular}{|l|}
2.17 \\
\end{tabular} & 0.14 & \begin{tabular}{|l|} 
\\
\end{tabular} & 99.1 \\
\hline EYB15 & 63.10 & 0.64 & 16.02 & 5.62 & 0.11 & 2.31 & \begin{tabular}{|l|}
5.33 \\
\end{tabular} & 3.63 & \begin{tabular}{|l|}
1.94 \\
\end{tabular} & 0.14 & \begin{tabular}{|l|}
0.90 \\
\end{tabular} & 9.7 \\
\hline 24 & .18 & 0.52 & 17.21 & 5.19 & 0.11 & 1.80 & 4.48 & 4.80 & \begin{tabular}{|l|}
1.49 \\
\end{tabular} & 0.12 & 2.80 & 99. \\
\hline 30 & 13 & 0.79 & 17.05 & 7.25 & 0.14 & 3.41 & 6.72 & 3.65 & \begin{tabular}{|l|}
1.66 \\
\end{tabular} & 0.18 & 0.70 & 99.1 \\
\hline YB34 & 0.73 & 0.66 & 16.72 & 6.28 & 0.13 & 2.40 & 5.33 & 3.76 & \begin{tabular}{|l|}
2.09 \\
\end{tabular} & 0.15 & 1.40 & 99. \\
\hline EYB35 & 61.80 & 0.58 & 16.52 & 5.69 & 0.12 & 2.22 & 5.05 & 3.64 & \begin{tabular}{|l|}
2.40 \\
\end{tabular} & 0.14 & \begin{tabular}{|l|}
1.50 \\
\end{tabular} & 99. \\
\hline EYB38 & 61.19 & 0.66 & 16.52 & 6.14 & 0.12 & 2.60 & 5.67 & 3.67 & 2.01 & 0.15 & 1.00 & 99.7 \\
\hline KOZ1 & 66.01 & 0.42 & 16.09 & 3.61 & 0.06 & 1.58 & 3.50 & 3.62 & \begin{tabular}{|l|}
3.40 \\
\end{tabular} & 0.16 & 1.20 & 99. \\
\hline KOZ2 & 3.04 & 0.53 & 16.08 & 4.32 & 0.07 & 2.29 & \begin{tabular}{|l|}
4.38 \\
\end{tabular} & 3.47 & \begin{tabular}{|l|}
3.58 \\
\end{tabular} & 0.22 & 1.70 & 99.1 \\
\hline $\mathrm{OZ4}$ & .60 & 0.51 & 15.62 & 4.02 & 0.07 & 2.27 & 4.05 & 3.35 & \begin{tabular}{|l|}
3.77 \\
\end{tabular} & ( & 1.10 & 99. \\
\hline OZ5 & .44 & 0.29 & 14.47 & 2.14 & 0.05 & 0.64 & 2.16 & 3.59 & \begin{tabular}{|l|}
4.15 \\
\end{tabular} & 0.09 & 0.60 & 99. \\
\hline KOZ8 & 5.32 & 0.51 & 15.73 & 4.00 & 0.07 & 2.18 & \begin{tabular}{|l|}
3.98 \\
\end{tabular} & 3.41 & \begin{tabular}{|l|}
3.84 \\
\end{tabular} & 0.21 & \begin{tabular}{|l|}
0.40 \\
\end{tabular} & 99.6 \\
\hline KOZ9 & 64.19 & 0.50 & 16.18 & 4.14 & 0.07 & 2.21 & 4.16 & 3.53 & \begin{tabular}{|l|}
3.90 \\
\end{tabular} & 0.22 & 0.50 & 99.6 \\
\hline KOZ10 & 65.63 & 0.49 & 15.37 & 3.94 & 0.07 & 2.14 & 3.83 & 3.27 & \begin{tabular}{|l|}
3.85 \\
\end{tabular} & 0.21 & \begin{tabular}{|l|}
0.80 \\
\end{tabular} & 99.6 \\
\hline EVC1 & 61.99 & 0.57 & 16.73 & 5.78 & 0.10 & 2.41 & 4.95 & 3.36 & \begin{tabular}{|l|}
2.83 \\
\end{tabular} & 0.16 & 0.80 & 99.6 \\
\hline $\mathrm{VC} 2$ & 4.06 & 0.49 & 15.94 & 4.90 & 0.11 & 1.95 & 4.47 & 3.37 & \begin{tabular}{|l|}
2.87 \\
\end{tabular} & 0.13 & 1.50 & 99. \\
\hline $\mathrm{VC} 3$ & 3.68 & 0.50 & 16.40 & 5.04 & 0.11 & 1.94 & 4.71 & 3.50 & \begin{tabular}{|l|}
2.76 \\
\end{tabular} & 0.12 & 1.00 & 99. \\
\hline VC5 & 65.38 & 0.44 & 15.43 & 4.28 & 0.10 & 1.94 & \begin{tabular}{|l|}
4.03 \\
\end{tabular} & 3.22 & \begin{tabular}{|l|}
3.77 \\
\end{tabular} & 0.17 & 0.90 & 99 . \\
\hline EVC6 & 64.42 & 0.45 & 15.67 & 4.50 & 0.10 & 2.02 & \begin{tabular}{|l|}
4.39 \\
\end{tabular} & 3.27 & \begin{tabular}{|l|}
3.45 \\
\end{tabular} & 0.18 & 1.20 & 99. \\
\hline
\end{tabular}




\begin{tabular}{|c|c|c|c|c|c|c|c|c|c|c|c|c|}
\hline$\sqrt{\mathrm{C} 8}$ & \begin{tabular}{l|l}
.69 \\
\end{tabular} & 0.41 & 15.12 & 1.69 & 0.06 & 2.14 & 4.80 & 4.0 & 0.47 & 0.16 & 4.20 & \\
\hline RH1 & .47 & 0.39 & 17.24 & 4.60 & 0.09 & 1.80 & 5.39 & 3.74 & 2.17 & 0.10 & 0.67 & 99.66 \\
\hline RH3 & .81 & 0.38 & 17.44 & 4.29 & 0.09 & 1.66 & 5.16 & 4.01 & 1.98 & 0.12 & 0.71 & \\
\hline DRH5 & .50 & 0.32 & 17.05 & 3.44 & 0.08 & 1.36 & 4.80 & 3.94 & 2.05 & 0.10 & 0.93 & 99. \\
\hline RH6 & .93 & 0.37 & 16.59 & 2.76 & 0.06 & 0.77 & 2.00 & 4.77 & 6.42 & .12 & 1.08 & \\
\hline AP42 & .61 & 0.23 & 14.60 & 2.06 & 0.07 & 0.51 & 2.57 & 3.71 & 3.10 & 0.04 & 0.81 & \\
\hline KAP43* & 71.54 & 0.19 & 14.21 & 1.99 & 0.08 & 0.51 & 2.26 & 3.39 & 3.38 & 0.06 & 1.78 & \\
\hline KAP45 & 4.18 & 0.50 & 16.83 & 4.78 & 0.10 & 1.84 & 5.12 & 3.54 & 2.15 & 0.10 & 0.48 & \\
\hline KAP46 & 63.43 & 0.51 & 16.19 & 4.76 & 0.09 & 2.25 & 4.83 & 3.43 & 3.16 & 0.16 & 0.80 & \\
\hline KAP47* & 63.30 & 0.61 & 16.15 & 5.43 & 0.13 & 2.01 & 5.11 & 3.14 & 2.12 & 0.11 & 0.85 & 98 \\
\hline KAP52* & 69.17 & 0.27 & 16.02 & 2.43 & 0.07 & 0.53 & 3.41 & 4.39 & 2.30 & 0.07 & 0.62 & 99. \\
\hline AM28* & 71.99 & 0.21 & 14.35 & 1.86 & 0.06 & 0.59 & 1.77 & 3.29 & 4.12 & 0.09 & 1.21 & 99. \\
\hline CAM29* & 68.63 & 0.31 & 15.40 & 2.91 & 0.04 & 1.01 & 2.61 & 2.52 & 5.07 & 0.17 & 0.64 & 99. \\
\hline CAM30* & 65.20 & 0.44 & 16.66 & 4.00 & 0.05 & 1.50 & 3.75 & 3.68 & 3.26 & 0.25 & 0.80 & \\
\hline TOP9 & 64.55 & 0.38 & 16.59 & 4.34 & 0.14 & 1.40 & 5.26 & 3.77 & 1.88 & 0.12 & 0.67 & \\
\hline TOP11 & 66.49 & 0.34 & 16.83 & 3.67 & 0.11 & 0.99 & 4.93 & 3.99 & 1.73 & 0.11 & 0.44 & \\
\hline TOP12 & 67.37 & 0.29 & 16.44 & 3.32 & 0.10 & 1.05 & 4.37 & 3.38 & 2.71 & 0.08 & 0.70 & \\
\hline TPL1 & 6 & 0.60 & 16.79 & 5.73 & 0.13 & 2.15 & 5.41 & 4.18 & 1.89 & 0.16 & 0.92 & \\
\hline TPL13* & 70.20 & 0.28 & 14.51 & 2.60 & 0.08 & 0.92 & 2.73 & 4.27 & 3.50 & 0.05 & 0.61 & 99 \\
\hline ГPL14 & 54.94 & 0.76 & 17.37 & 7.39 & 0.15 & 4.52 & 8.63 & 3.42 & 1.19 & 0.16 & 0.89 & \\
\hline GÜR18* & 64.00 & 0.48 & 16.00 & 4.89 & 0.12 & 1.99 & 4.97 & 3.77 & 2.40 & 0.10 & 1.13 & \\
\hline GÜR19 & \begin{tabular}{|l|l|}
64.23 \\
\end{tabular} & 0.38 & 17.24 & 4.58 & 0.11 & 1.56 & 5.14 & 3.72 & 1.96 & 0.12 & 0.70 & \\
\hline SÜR20* & 64.10 & 0.42 & 16.38 & 4.47 & 0.12 & 1.70 & 4.96 & 3.70 & 1.97 & 0.13 & 1.13 & 99 \\
\hline GR23 & 66.72 & $\begin{array}{l}0.53 \\
\end{array}$ & 15.62 & 4.03 & 0.09 & 1.35 & 3.51 & 3.52 & 3.53 & 0.15 & 0.70 & 99 \\
\hline GR24* & \begin{tabular}{|c|}
69.73 \\
\end{tabular} & 0.36 & 14.57 & 2.68 & 0.06 & 0.82 & 2.29 & 4.06 & 3.97 & 0.10 & 0.77 & 99 \\
\hline GR27* & 67.84 & 0.45 & 15.18 & 3.17 & 0.07 & 1.03 & 2.94 & 3.45 & 3.89 & 0.12 & 1.06 & \\
\hline
\end{tabular}

\subsection{Rock-types and mineralogical composition}

As shown in Fig. 2, a variety of rock-types, from quartz monzodiorite to syenogranite has been studied. These may contain hornblende, biotite and muscovite as major mineral phases. The accessory minerals present are zircon, apatite, titanite, allanite and epidote.

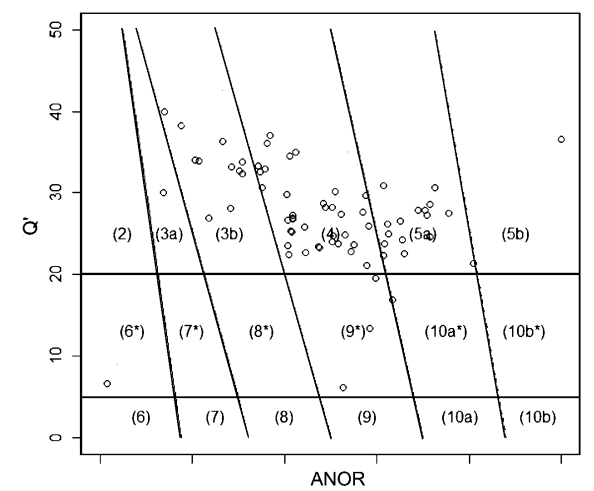

Figure 2 - Q'ANOR diagram (Streckeisen and Le Maitre, 1979) showing the classification of the samples. (2 Alkali-feldspar granite, 3a Syenogranite, 3b Monzogranite, 4 Granodiorite, 5a Tonalite, 5b Calcic Tonalite, 6* Alkali-feldspar Quartz-Syenite, 7* Quartz Syenite, 8* Quartz Monzonite, 9* Quartz Monzodiorite, 10a* Quartz diorite, 10b* Quartz Gabbro, 6 Alkali-feldspar Syenite, 7 Syenite, 8 Monzonite, 9 Monzogabbro, 10a Diorite, 10b Gabbro. 


\section{Results}

\subsection{Radiation indices and dose estimations}

For each pluton of the Western Anatolia studied, the average values of the specific activities of ${ }^{226} \mathrm{Ra}$, ${ }^{232} \mathrm{Th}$ and $\left.{ }^{40} \mathrm{~K}_{(\mathrm{Bq} \mathrm{kg}}{ }^{-1}\right)$, the external gamma index, the internal alpha index and the effective dose rate $\left(\mathrm{mSv}^{-1}\right)$ received indoors and outdoors due to the usage of the studied samples as decorative building materials are given in Table 2 .

Table 2 - ${ }^{226} \mathrm{Ra},{ }^{232} \mathrm{Th}$ and ${ }^{40} \mathrm{~K}\left(\mathrm{~Bq} \cdot \mathrm{kg}^{-1}\right), \mathrm{I}_{\gamma}, \mathrm{I}_{a}, \mathrm{H}_{\text {ext }}$ and $\mathrm{H}_{\text {int }}\left(\mathrm{mSv} \cdot \mathrm{y}^{-1}\right)$ values.

\begin{tabular}{|c|c|c|c|c|c|c|c|c|}
\hline & & ${ }^{226} \mathrm{Ra}$ & ${ }^{232} \mathrm{Th}$ & ${ }^{40} \mathrm{~K}$ & $\overline{\mathrm{I}_{\gamma}}$ & $\mathrm{I}_{\alpha}$ & $\mathrm{H}_{\mathrm{ext}}$ & $\overline{\mathrm{H}_{\text {int }}}$ \\
\hline AVERAGE & \multirow{4}{*}{ Ilica } & 67.05 & 106.19 & 1210.22 & 1.16 & 0.34 & 0.33 & 0.17 \\
\hline MIN & & 33.01 & 0.12 & 854.58 & 0.40 & 0.17 & 0.16 & 0.09 \\
\hline MAX & & 100.58 & 185.66 & 1998.43 & 1.93 & 0.50 & 0.49 & 0.26 \\
\hline $\mathrm{N}$ & & \multicolumn{7}{|c|}{10} \\
\hline AVERAGE & \multirow{4}{*}{ Çataldağ } & 108.18 & 101.16 & 1344.76 & 1.31 & 0.54 & 0.53 & 0.28 \\
\hline MIN & & 39.07 & 23.76 & 772.27 & 0.51 & 0.20 & 0.19 & 0.10 \\
\hline MAX & & 229.62 & 158.98 & 1894.11 & 2.19 & 1.15 & 1.13 & 0.60 \\
\hline $\mathrm{N}$ & & \multicolumn{7}{|c|}{8} \\
\hline AVERAGE & \multirow{4}{*}{ Uludağ } & 74.86 & 77.80 & 1072.50 & 1.00 & 0.37 & 0.37 & 0.19 \\
\hline MIN & & 57.37 & 63.90 & 914.15 & 0.82 & 0.29 & 0.28 & 0.15 \\
\hline MAX & & 92.96 & 99.30 & 1235.47 & 1.22 & 0.46 & 0.46 & 0.24 \\
\hline $\mathrm{N}$ & & \multicolumn{7}{|c|}{6} \\
\hline AVERAGE & \multirow{4}{*}{ Eybek } & 35.48 & 53.51 & 680.78 & 0.61 & 0.18 & 0.17 & 0.09 \\
\hline MIN & & b.d.l. & 35.40 & 462.73 & 0.43 & 0.14 & 0.14 & \\
\hline MAX & & 42.93 & 70.10 & 811.71 & 0.76 & 0.21 & 0.21 & 0.11 \\
\hline $\mathrm{N}$ & & \multicolumn{7}{|c|}{7} \\
\hline AVERAGE & \multirow{4}{*}{ Kozak } & 59.95 & 106.26 & 1267.88 & 1.15 & 0.30 & 0.29 & 0.16 \\
\hline MIN & & b.d.l. & 87.12 & 1153.13 & 0.98 & 0.24 & 0.24 & \\
\hline MAX & & 86.31 & 131.11 & 1498.80 & 1.44 & 0.43 & 0.42 & 0.22 \\
\hline $\bar{N}$ & & \multicolumn{7}{|c|}{6} \\
\hline AVERAGE & \multirow{4}{*}{ Evciler } & 61.92 & 107.00 & 874.26 & 1.03 & 0.31 & 0.30 & 0.16 \\
\hline MIN & & b.d.l. & 70.52 & 141.97 & 0.54 & 0.21 & 0.21 & - \\
\hline MAX & & 83.96 & 135.43 & 1261.58 & 1.38 & 0.42 & 0.41 & 0.22 \\
\hline $\mathrm{N}$ & & \multicolumn{7}{|c|}{6} \\
\hline AVERAGE & \multirow{4}{*}{ Orhaneli } & 40.87 & 74.26 & 1192.70 & 0.91 & 0.20 & 0.20 & 0.11 \\
\hline MIN & & 15.26 & 27.57 & 704.81 & 0.42 & 0.08 & 0.07 & 0.04 \\
\hline MAX & & 116.58 & 207.32 & 2541.95 & 2.27 & 0.58 & 0.57 & 0.30 \\
\hline $\mathrm{N}$ & & \multicolumn{7}{|c|}{4} \\
\hline AVERAGE & \multirow{4}{*}{ Kapıdağ } & 28.40 & 38.66 & 885.71 & 0.58 & 0.14 & 0.14 & 0.07 \\
\hline MIN & & 11.76 & 14.13 & 372.76 & 0.23 & 0.06 & 0.06 & 0.03 \\
\hline MAX & & 61.75 & 57.59 & 1385.18 & 0.96 & 0.31 & 0.30 & 0.16 \\
\hline $\mathrm{N}$ & & \multicolumn{7}{|c|}{6} \\
\hline AVERAGE & \multirow{4}{*}{ Çamlık } & 66.54 & 67.62 & 1338.40 & 1.01 & 0.33 & 0.33 & 0.17 \\
\hline MIN & & b.d.l. & 59.61 & 1011.72 & 0.86 & 0.33 & 0.33 & - \\
\hline MAX & & 66.54 & 73.82 & 1597.66 & 1.12 & 0.33 & 0.33 & 0.17 \\
\hline $\mathrm{N}$ & & \multicolumn{7}{|c|}{3} \\
\hline AVERAGE & \multirow{3}{*}{ Topuk } & 37.48 & 56.62 & 834.54 & 0.69 & 0.19 & 0.18 & 0.10 \\
\hline MIN & & 18.46 & 36.16 & 647.67 & 0.46 & 0.09 & 0.09 & 0.05 \\
\hline MAX & & 56.51 & 71.35 & 956.47 & 0.86 & 0.28 & 0.28 & 0.15 \\
\hline
\end{tabular}




\begin{tabular}{|c|c|c|c|c|c|c|c|c|}
\hline & & ${ }^{226} \mathrm{Ra}$ & ${ }^{232} \mathrm{Th}$ & ${ }^{40} \mathrm{~K}$ & $\overline{\mathrm{I}_{\gamma}}$ & $\overline{\mathrm{I}_{\alpha}}$ & $\mathrm{H}_{\mathrm{ext}}$ & $\overline{\mathrm{H}_{\mathrm{int}}}$ \\
\hline $\mathrm{N}$ & & \multicolumn{7}{|c|}{3} \\
\hline AVERAGE & \multirow{4}{*}{ Tepeldağ } & 16.75 & 79.40 & 1180.81 & 0.85 & 0.08 & 0.08 & 0.04 \\
\hline MIN & & b.d.l. & 18.05 & 414.65 & 0.27 & 0.06 & 0.05 & 0.03 \\
\hline MAX & & 22.49 & 143.81 & 1768.80 & 1.38 & 0.11 & 0.11 & 0.06 \\
\hline $\mathrm{N}$ & & \multicolumn{7}{|c|}{3} \\
\hline AVERAGE & Gürgenyayla & 23.69 & 33.07 & 662.47 & 0.47 & 0.12 & 0.12 & 0.06 \\
\hline MIN & & 21.64 & 19.96 & 532.84 & 0.35 & 0.11 & 0.11 & 0.06 \\
\hline MAX & & 25.74 & 41.10 & 863.33 & 0.58 & 0.13 & 0.13 & 0.07 \\
\hline $\mathrm{N}$ & & \multicolumn{7}{|c|}{3} \\
\hline AVERAGE & \multirow{4}{*}{ Ĕ̆rigöz } & 41.64 & 76.66 & 1346.05 & 0.97 & 0.21 & 0.20 & 0.11 \\
\hline MIN & & 33.93 & 66.55 & 1205.92 & 0.85 & 0.17 & 0.17 & 0.09 \\
\hline MAX & & 49.15 & 95.08 & 1484.57 & 1.13 & 0.25 & 0.24 & 0.13 \\
\hline $\mathrm{N}$ & & \multicolumn{7}{|c|}{3} \\
\hline
\end{tabular}

According to the experimental results, the natural radioactivity levels were ranged up to 229.62Bq. $\mathrm{kg}^{-1}$ for ${ }^{226} \mathrm{Ra}$, up to $207.32 \mathrm{~Bq} \cdot \mathrm{kg}^{-1}$ for ${ }^{232} \mathrm{Th}$ and up to $2541.95 \mathrm{~Bq} \cdot \mathrm{kg}^{-1}$ for ${ }^{40} \mathrm{~K}$, with a mean value of $57.67( \pm 38.13), 80.30( \pm 42.00)$ and $1071.92( \pm 405.24) \mathrm{Bq} \mathrm{kg}^{-1}$ respectively. Comparing the activities of ${ }^{226} \mathrm{Ra}$ and ${ }^{232} \mathrm{Th}$ of the samples analyzed with the average granite concentrations (UNSCEAR 2000), it can be seen that the activities of the majority of the samples studied are below the average values of 78 and $111 \mathrm{~Bq} \cdot \mathrm{kg}^{-1}$ in most cases (Table 2). Consequently, the granites studied are be competitive to the commercial granites worldwide. A radiological study, concerning radiation index and dose estimation is required in order to strengthen the above conclusion.

Aiming to protect the public from excessive exposure to radioactivity, various radioactivity indices have been proposed in order to assess the natural radioactivity of building materials. Radionuclides in building materials are the sources of both external exposure due to gamma-rays emitted by ${ }^{40} \mathrm{~K}$, ${ }^{226} \mathrm{Ra}$ and ${ }^{232} \mathrm{Th}$ as well as internal exposure caused by alpha-particles deposited on the respiratory tract tissues due to inhalation of radon indoors. Indoors environment is generally described by a standard room model. Three typical room models have been adopted up to now, (a) a parallelepiped room $(4 \times 5 \times 2.8 \mathrm{~m})$ with wall density $2350 \mathrm{~kg} \cdot \mathrm{m}^{-3}$ and thickness $0.2 \mathrm{~m}$; (b) a spherical shell with radius $2.7 \mathrm{~m}$, peripheral thickness $0.223 \mathrm{~m}$ and density $1890 \mathrm{~kg} \cdot \mathrm{m}^{-3}$, and (c) a hole surrounded by an infinite thickness medium (Krisiuk et al., 1971; Stranden, 1979; Koblinger, 1984). In the present study the indices adopted by the European Commission (E.C., 1999) were applied considering a standard parallelepiped room model with no doors and windows. Taking into account that the external exposure due to the building materials has a limit of $1 \mathrm{mSv} \cdot \mathrm{y}^{-1}$ then the following formula of external gamma index $\left(\mathrm{I}_{\gamma}\right)$ is calculated as:

$$
I_{\gamma}=\frac{C_{R a}}{300 B q \cdot k g^{-1}}+\frac{C_{T h}}{200 B q \cdot k g^{-1}} \frac{C_{K}}{3000 B q \cdot k g^{-1}}
$$

Materials having $\mathrm{I}_{\gamma}<2$ would increase the annual effective dose by $0.3 \mathrm{mSv}$, while for $2<\mathrm{I}_{\gamma}<6$, the gamma-ray index corresponds to an increase in effective dose by $1 \mathrm{mSv} \cdot \mathrm{y}^{-1}$. Building materials used superficially rather than in bulk amounts should be exempted from all restrictions concerning radioactivity, if the excess of gamma radiation originating from them increases the annual effective dose of a member of the public by $0.3 \mathrm{mSv}$ at the most. On the other hand, dose rates higher than $1 \mathrm{mSv} . \mathrm{y}^{-1}$ are allowed only in exceptional cases, where materials are locally used. Finally, samples with $\mathrm{I}_{\gamma}>6$ cannot be recommended for use in buildings (E.C., 1999). In case of internal alpha radiation exposure the following formula has been applied, taking into consideration that a building material with ${ }^{226} \mathrm{Ra}$ concentration lower than $200 \mathrm{~Bq} \cdot \mathrm{kg}^{-1}$ could not cause indoor radon concentration higher than $200 \mathrm{~Bq} \cdot \mathrm{m}^{-3}$, which is the recommended action level of indoor radon exposure by EU and ICRP for dwellings (E.C., 1990; ICRP, 1994; Righi and Bruzzi, 2006). 


$$
I_{\alpha}=\frac{C_{R a}}{200 B q \cdot k^{-1}} \leq 1
$$

The index factors estimated above, correspond to a standard room with massive granitic walls and could be applied more to workers in a well-ventilated granite mine than inhabitants. For the estimation of the actual dose received annually indoors, due to granite tiles usage, a more realistic case has to be considered where granite tiles with $\sim 2 \mathrm{~cm}$ in thickness cover only the floor of the standard room (Anjos et al., 2005, 2011; Salas et al., 2006; Mao et al., 2006). In this case, the absorbed gamma dose rate $\left(D_{a}, n G y \cdot h^{-1}\right)$, denoted as the energy transfer rate by ionizing radiation absorbed per unit mass of the tissue, due to granite floor could be calculated as:

$$
D_{a}\left(n G y \cdot h^{-1}\right)=0.172 \cdot C_{R a}+0.217 \cdot C_{T h}+0.015 \cdot C_{K}
$$

where $\mathrm{C}_{\mathrm{Ra}}, \mathrm{C}_{\mathrm{Th}}$ and $\mathrm{C}_{\mathrm{K}}$ are the activity concentrations $\left(\mathrm{Bq} \cdot \mathrm{kg}^{-1}\right)$ of ${ }^{226} \mathrm{Ra},{ }^{232} \mathrm{Th}$ and ${ }^{40} \mathrm{~K}$ in the samples. Then, taking into account the indoor occupancy factor $\left(\mathrm{T}, 7000 \mathrm{~h} . \mathrm{y}^{-1}\right)$, which implies that $80 \%$ of time is spent indoors, and the doses conversion factor $\left(\mathrm{F}, 0.7 \mathrm{~Sv}^{-G y}{ }^{-1}\right)$, the increment of the effective dose rate due to gamma radiation received indoors derives as follows:

$$
H_{e x t}\left(m S v \cdot y^{-1}\right)=10^{-6} \cdot\left(0.7 \cdot C_{R a} \cdot 7000\right)
$$

The effective dose rate due to radon exposure indoors is estimated as:

$$
H_{\text {int }}\left(m S v \cdot y^{-1}\right)=10^{-3} \cdot\left(f_{P-e q} \cdot D_{c} \cdot B \cdot F \cdot C_{R n}\right)
$$

where $C_{R n}$ is the radon concentration indoors $\left(\mathrm{Bq} \cdot \mathrm{m}^{-3}\right), F$ is the appropriate equilibrium factor between radon and its daughters, $f_{p-e q}$ is the conversion factor from equilibrium equivalent radon concentration $\left(F \cdot C_{R n}\right)$ to potential alpha energy concentration $\left(5.56 .10^{-9} \mathrm{~J}^{-\mathrm{m}^{-3}}\right.$ per Bq. $\left.\mathrm{m}^{-3}\right), D_{c}$ is the conversion factor from potential alpha energy concentration to the effective dose $(2 \mathrm{~Sv} / \mathrm{J})$, and $B$ is the annual breathing rate $\left(7013 \mathrm{~m}^{3} \cdot \mathrm{y}^{-1}\right)$. For a well ventilated room the equilibrium factor $\mathrm{F}$ ranges from 0.5 to 0.7 , hence using equation (5) results in $1 \mathrm{~Bq} \cdot \mathrm{m}^{-3}$ of radon which corresponds to an effective dose rate 0.039 - $0.055 \mathrm{mSv} . \mathrm{y}^{-1}$ due to alpha radiation (ICRU, 1994; E.C., 1990).

The radon concentrations indoor due to radon exhalation from the granitic floor existing in the room can be determined by the following formula:

$$
C_{R n}\left(B q \cdot m^{-3}\right)=\frac{(1 / 2) \cdot C_{R a} \cdot \varepsilon \cdot \lambda \cdot \rho \cdot d \cdot S}{V \cdot\left(\lambda_{v}+\lambda\right)}
$$

Considering the parallelepiped standard room with ventilation rate $\lambda_{v}=1 \mathrm{~h}^{-1}$ (that corresponds to an equilibrium factor $\mathrm{F}=0.7)$ and the floor covered by granite tiles with $1.5 \mathrm{~cm}$ in thickness (d), $2650 \mathrm{~kg} \cdot \mathrm{m}^{-3}$ density $(\rho)$ and $8 \%$ emanation factor $(\varepsilon)$ as representative values, the internal effective dose rate is calculated as: (Bruzzi et al., 1992; Stoulos et al., 2003; Anjos et al., 2011):

$$
H_{\text {int }}\left(m S v \cdot y^{-1}\right)=0.0026 \cdot C_{R a}
$$

The range, standard deviation, standard error, average and median values of $\mathrm{I}_{\gamma}, \mathrm{I}_{\alpha}, \mathrm{H}_{\mathrm{ext}}$ and $\mathrm{H}_{\text {int }}$ for each of the Western Anatolian plutons studied are given in Fig. 2.

\section{Conclusions}

The excess on the effective dose received annually indoors due to granite tiles usage is estimated considering a standard room model where granite tiles with few $\mathrm{cm}$ in thickness cover only the floor of the room. The increment on the external $\gamma$-radiation effective dose rate appears a mean value of $0.27( \pm$ $0.19) \mathrm{mSv} \cdot \mathrm{y}^{-1}$, scattering well below $1 \mathrm{mSv} \cdot \mathrm{y}^{-1}$. In case of the internal $\alpha$-radiation a mean value of 0.14 $( \pm 0.10) \mathrm{mSv} . \mathrm{y}^{-1}$, scattering below $0.5 \mathrm{mSv} \cdot \mathrm{y}^{-1}$ has been found. The majority of the granite samples increase the external as well as the internal dose less than $30 \%$ of the maximum permitted limit of the effective dose rate. Only one sample from Cataldag pluton seems to exceed the effective dose received outdoors and indoors. Moreover, Cataldag pluton shows the highest average activities of radionuclides and thus, values of radioactive indices. Therefore, at least from radiological point of view, the majority of granitic rocks studied could be safely used as decorative building materials. 


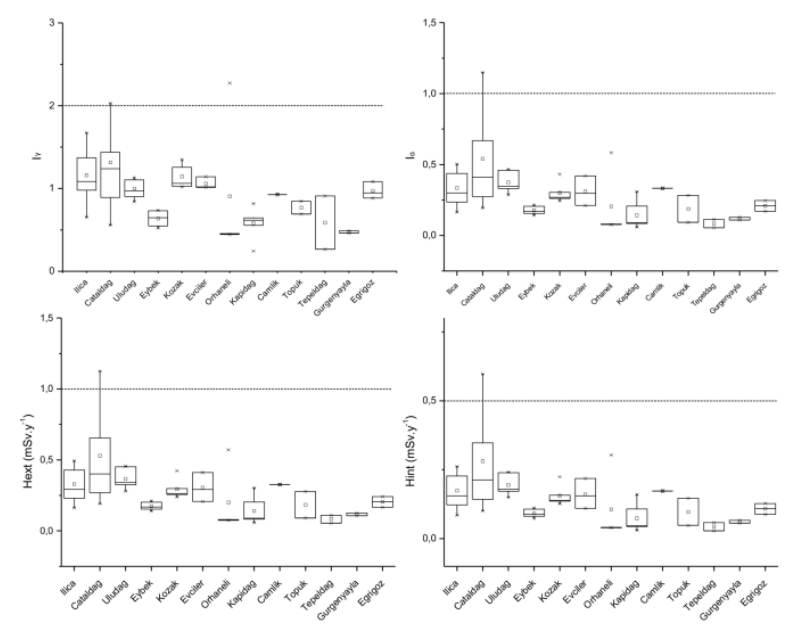

Figure $2-I_{\gamma}, I_{\alpha}, H_{e x t}$ and $H_{\text {int }}$ values of the samples studied for each Western Anatolian pluton. (The box corresponds to the standard error while the whisker to the standard deviation). $X$ : max and min values, black star: mean value, dashed lines: permitted limits.

\section{Acknowledgments}

This study has been funded by grants from the Istanbul Technical University (BAP Project No: 37883 and 36010) and the Turkish Research Council (TUBITAK-CAYDAG-112Y093) that are gratefully acknowledged.

\section{References}

Akay, E., 2009. Geology and Petrology of the Simav Magmatic Complex (NW Anatolia) and its comparison with the Oligo-Miocene granitoids in NW Anatolia: implications on Tertiary tectonic evolution of the region, Int. J. Earth Sci. (GeolRundsch), 98, 1655-1675.

Aldanmaz, E., Pearce, J., Thirlwall, M.F. and Mitchell, J., 2000. Petrogenetic evolution of late Cenozoic, post-collision volcanism in western Anatolia, Turkey, J. Volcanol. Geoth. Res., 102, 67-95.

Altunkaynak, Ş., 2007. Collision-driven slab breakoff magmatism in northwestern Anatolia, Turkey, J. Geol., 115, 63-82.

Altunkaynak, Ş. and Yılmaz, Y., 1998. The Kozak magmatic complex; western Anatolia, J. Volcanol. Geoth. Res., 85/1-4, 211-231.

Altunkaynak, Ş. and Dilek, Y., 2006. Timing and nature of post collisional volcanism in Western Anatolia and Geodynamic Implications. In: Dilek, Y. and Pavlides, S., eds., Post-Collisional Tectonics and Magmatism of the Eastern Mediterranean Region, Geological Society of America Special Paper, 409, 321-351.

Altunkaynak, Ş. and Genç, Ş.C., 2008. Petrogenesis and time-progressive evolution of the Cenozoic continental volcanism in the Biga Peninsula, NW Anatolia (Turkey), Lithos, 102, 316-340.

Altunkaynak, S., Dilek, Y., Genç, S.C., Sunal, G., Gertisser, R., Furnes, H., Foland, K.A. and Yang, J., 2012a. Spatial, temporal and geochemical evolution of OligoMiocene granitoid magmatisin western Anatolia, Turkey, Gondwana Res., 21 (2012A), 961-986.

Altunkaynak, S., Sunal, G., Aldanmaz, E., Genç, S.C., Dilek, Y., Furnes, H., Foland, K.A., Yang, J. and Y1ldı, M., 2012b. Eocene Granitic Magmatism in NW Anatolia (Turkey) revisited: New implications from comparative zircon SHRIMP U-Pb and 40Ar-39Ar geochronology and isotope geochemistry on magma genesis and emplacement, Lithos, 155, 289-309.

Anjos, R.M, Veiga, R., Soares, T., Santos, A.M.A, Aguiar, J.G., Frasca, M.H.B.O., Brage, J.A.P., Uzeda, D., Mangia, L., Facure, A., Mosquera, B., Carvalho, C. and Gomes, P.R.S., 2005. Natural radionuclide distribution in Brazilian commercial granites, Rad. Measur., 39, 245-253. 
Anjos, R.M., Ayub, J.J., Cid, A.S., Cardoso, R. and Lacerda, T., 2011. External gamma-ray dose rate and radon concentration in indoor environments covered with Brazilian granites, $J$. Envir. Radioact., 102,1055-1061.

Boztuğ, D., Harlavan, Y., Jonckheere, R., Can, I. and Sarı, R., 2009. Geochemistry and K-Ar cooling ages of the Ilıca, Cataldag (Balıkesir) and Kozak (Izmir) granitoids, west Anatolia, Turkey.

Dilek, Y. and Altunkaynak, Ş., 2007. Cenozoic crustal evolution and mantle dynamics of postcollisional magmatism in western Anatolia, Int. Geol. Rev., 49(5), 431-453.

Erkül, F., 2010. Tectonic significance of synextensional ductile shear zones within the Early Miocene Alaçamdağ granites, northwestern Turkey, Geol. Mag., 147, 611-637.

Erkül, S.T. and Erkül, F., 2012. Magma interaction processes in syn-extensional granitoids: The Teriary Menderes Metamorphic Core Complex, western Turkey, Lithos, 142-143, 16-33.

Ersoy, Y.E., Helvac1, C. and Palmer, M.R. Petrogenesis of the Neogene volcanic units in the NE-SWtrending basins in western Anatolia, Turkey, Contrib. to Mine and Petrol., 163, 379-401.

European Commission (E.C.), 1999. Radiation Protection 112: Radiological Protection Principles Concerning the Natural Radioactivity of Building Materials Directorate -General Environment, Nuclear Safety and Civil Protection.

Genç, Ş.C. and Yılmaz, Y., 1997. An example of post-collisional magmatism in northwestern Anatolia: the Kizderbent Volcanics (Armutlu Peninsula, Turkey), Turk. J. Earth. Sci., 6, 33-42.

Güleç, N., 1991. Crust-mantle interaction in western Turkey: implications from $\mathrm{Sr}$ and $\mathrm{Nd}$ isotope geochemistry of Tertiary and Quaternary volcanics, Geol. Mag., 23, 417-435.

Harris, N.B.W., Kelley, S. and Okay, A.I., 1994. Post-collisional magmatism and tectonics in northwest Anatolia, Contrib. to Mine and Petrol., 117, 241-252.

Hasözbek, A., Satır, M., Erdoğan, B., Akay, E. and Siebel W., 2010. Early Miocene post-collisional magmatism in NW Turkey: geochemical and geochronological constraints, Int. Geol. Rev., 1-22.

Köprübaşı, N. and Aldanmaz, E., 2004. Geochemical constraints on the petrogenesis of Cenozoic I-type granitoids in Northwest Anatolia, Turkey: Evidence for magma generation by lithospheric delamination in a post-collisional setting, Int. Geol. Rev., 46, 705-729.

Mao, Y., Liu, Y., Fu, Y. and Lin, L., 2006. Physical models and limits of radionuclides for decorative building materials, Health Physics, 90, 471-476.

Okay, A.I. and Satır, M., 2000. Coeval plutonism and metamorphism in a latest Oligocene metamorphic core complex in northwest Turkey, Geol. Mag., 137, 495-516.

Okay, A.I. and Satır, M., 2006. Geochronology of Eocene plutonism and metamorphism in northwest Turkey: evidence for a possible magmatic arc, Geodin. Acta, 19(5), 251-266.

Özgenç, İ. and İlbeyli, N., 2008. Petrogenesis of the Late Cenozoic Eğrigöz Pluton in Western Anatolia, Turkey: implications for magma genesis and crustal processes, Int. Geol. Rev., 50, 375-391.

Papadopoulos, A., Christofides, G., Koroneos, A., Papadopoulou, L., Papastefanou, C. and Stoulos, S., 2013. Natural radioactivity and radiation index of the major granitic plutons in Greece, $J$. Envir. Radioact., 124, 227-238.

Streckeisen, A. and Le Maitre, R.W., 1979. A chemical approximation to the modal QAPF classification of the igneous rocks, Egypt, Neues Jahrb. Mineral Abh., 136, 169-206.

Salas, H.T, Nalini Jr, H.A. and Mendes, J.C., 2006. Radioactivity dosage evaluation of Brazilian ornamental granitic rocks bases on chemical data, with mineralogical and lithological characterization, J. Radioanal. Nucl. Chem., 267, 669-673.

Şengör, A.M.C. and Y1lmaz, Y., 1981. Tethyan evolution of Turkey: a plate tectonic approach, Tectonophysics, 75, 181-241.

UNSCEAR, 2000. United Nations Scientific Committee on the Effects of Atomic Radiation. Sources and Effects of Ionising Radiation, Vol. I. United Nations, New York.

Y1lmaz, Y., 1989. An approach to the origin of young volcanic rocks of western Turkey. In: Şengör, A. M. C., eds., Tectonic evolution of the Tethyan region: The Hague, Kluwer Academic, 159-189.

Yılmaz, Y., Genç, Ş.C., Gürer, O.F., Bozcu, M., Yılmaz, K., Karacik, Z., Altunkaynak, Ş. and Elmas, A., 2000. When did the western Anatolian grabens begin to develop? In: Bozkurt, E., Winchester, J.A. and Piper, J.A.D., eds., Tectonics and Magmatism in Turkey and the Surrounding Area, Geological Society, London, Special Publication, 173, 353-384. 